\title{
Prescribing patterns for attention deficit hyperactivity disorder medications among children and adolescents in Korea, 2007-2011
}

\author{
Inmyung Song ${ }^{1}$, Ju-Young Shin ${ }^{2}$ \\ 'Division of Risk Assessment and International Cooperation, Centers for Disease Control and Prevention, Cheongju; ${ }^{2}$ School of Pharmacy, \\ Sungkyunkwan University, Suwon, Korea
}

OBJECTIVES: This study analyzed the prevalence of attention deficit hyperactivity disorder (ADHD) medication use among children and adolescents in Korea between January 1, 2007 and December 31, 2011.

METHODS: Using the Korea National Health Insurance claims database, we identified patients between one and 17 years of age who had at least one medical claim for the diagnosis of ADHD (International Classification of Diseases, 10th revision: F90.0). The annual prevalence of ADHD diagnoses was calculated, using national census data from Statistics Korea on the population aged between one and 17 years as the denominator. The prevalence was age-standardized using the 2010 population as the standard population. The number of patients who were treated with methylphenidate and/or atomoxetine and the prevalence of total patients with ADHD that were treated with either drug were also calculated for each year. All analyses were stratified according to gender and age group (1-5 years, 6-12 years, and 13-17 years).

RESULTS: The number of patients diagnosed with ADHD increased from 72,704 persons $(0.71 \%)$ in 2007 to 85,468 persons $(0.93 \%)$ in 2011 . The annual age-standardized prevalence of ADHD diagnoses increased from $0.67 \%$ in 2007 to $0.94 \%$ in 2011 . The prevalence of methylphenidate use among children and adolescents with ADHD decreased from $73.91 \%$ in 2007 to $70.33 \%$ in 2011, whereas that of atomoxetine use increased from $5.77 \%$ in 2009 to $13.09 \%$ in 2011.

CONCLUSIONS: While methylphenidate remains the most commonly prescribed ADHD drug, the use of atomoxetine has increased.

KEY WORDS: Attention deficit hyperactivity disorder, Methylphenidate, Atomoxetine hydrochloride, Attention, Prescriptions

\section{INTRODUCTION}

Attention deficit hyperactivity disorder (ADHD) is the most common pediatric mental disorder, and the prevalence of $\mathrm{ADHD}$ is increasing in many parts of the world [1]. The use of ADHD

Correspondence: Ju-Young Shin

School of Pharmacy, Sungkyunkwan University, 2066 Seobu-ro, Jangan-gu, Suwon 16419 , Korea

Tel: +82-31-290-7702, Fax: +82-31-292-8800, E-mail: shin.jy@skku.edu

Received: Oct 4, 2016, Accepted: Oct 26, 2016, Published: Oct 26, 2016

This article is available from: http://e-epih.org/

(C) 2016, Korean Society of Epidemiology

(C) This is an open-access article distributed under the terms of the Creative Commons Attribution License (http://creativecommons.org/licenses/by/4.0/), which permits unrestricted use, distribution, and reproduction in any medium, provided the original work is properly cited. medications such as methylphenidate in the US and Europe has been extensively studied [2,3], but in Asia, it has only been studied in Hong Kong [4]. Most studies have reported dramatic increases in the use of ADHD medications.

It has been documented that methylphenidate has greater effects than atomoxetine on performance measures of sustained attention in youths with ADHD [5]. The rate of new atomoxetine use in the US decreased in all age groups even before the Food and Drug Administration issued a warning concerning suicidal thinking [6]. Since the approval of atomoxetine, a newer ADHD medication, in 2006, changes in patterns of prescribing ADHD medications in Korea have not been fully explored. This study aimed to investigate the prevalence of ADHD diagnoses and changing patterns in the prescription of methylphenidate and atomoxetine among children and adolescents with 
ADHD between January 1, 2007 and December 31, 2011.

\section{MATERIALS AND METHODS}

\section{Study subjects}

This study was conducted using the Korea National Health Insurance (NHI) claims database. The study subjects consisted of children and adolescents in Korea aged between one and 17 years who had been diagnosed with ADHD between January 1 , 2007 and December 31, 2011. We identified patients who had at least one medical claim containing an International Classification of Diseases, 10th revision code for ADHD (F90.0) as a primary or secondary diagnosis. The ADHD medications included methylphenidate and atomoxetine prescribed at least once in inpatient or outpatient settings. Methylphenidate and atomoxetine are the only drugs that have been approved for the treatment of ADHD in Korea; dexamphetamine has not been approved. All preparations of methylphenidate, such as immediate release and sustained release formulations, were included.

\section{Prevalence of attention deficit hyperactivity disorder diagnoses and attention deficit hyperactivity disorder medication use}

The annual prevalence of ADHD diagnoses was calculated using the national census data from Statistics Korea on the total population aged between one and 17 years as the denominator. The prevalence of ADHD diagnosis was age-standardized using the 2010 population as the standard population. The number and proportion of patients with ADHD who were treated with either methylphenidate or atomoxetine were also calculated for each year. The number of prescriptions of ADHD medications per year and the prescribed days of ADHD medication per pa- tient were also estimated. All analyses were stratified by gender and age group (1-5 years, 6-12 years, and 13-17 years).

To examine the time trend of prevalence, the monthly numbers of patients diagnosed with ADHD and patients treated with medications were calculated and plotted. Drug treatment groups were categorized into methylphenidate users, atomoxetine users, and combined users.

All statistical analyses were performed using SAS version 9.3 (SAS Institute Inc., Cary, NC, USA). The study protocol was approved by the institutional review board (IRB) of the Sungkyunkwan University (IRB no. 2016-08-013).

\section{RESULTS}

Overall, fewer than $1 \%$ of children and adolescents in Korea were diagnosed with ADHD in 2007-2011. The total number of patients diagnosed with ADHD increased from 72,704 persons $(0.71 \%)$ in 2007 to 85,468 persons $(0.93 \%)$ in 2011 . The age-standardized annual prevalence of ADHD diagnosis increased from $0.67 \%$ in 2007 to $0.94 \%$ in 2011 . The prevalence increased from $1.07 \%$ to $1.41 \%$ in boys and from $0.30 \%$ to $0.41 \%$ in girls over the study period. The prevalence increased from $1.09 \%$ to $1.45 \%$ in children $6-12$ years of age and from $0.58 \%$ to $0.94 \%$ in those $13-17$ years of age. The mean $( \pm$ standard deviation [SD]) age of the diagnosed patients increased from $10.41( \pm 3.18)$ years in 2007 to $11.19( \pm 3.20)$ years in 2011 . The mean age of patients on methylphenidate or atomoxetine increased as well. The number of diagnosed patients aged 1-5 years decreased from $2,944(0.13 \%)$ to $2,449(0.11 \%)$, while the number of patients 13-17 years of age diagnosed with ADHD increased from $20,316(0.58 \%)$ to $31,413(0.94 \%)$ between 2007 and 2011 (Table 1).

Table 1. Age-standardized annual prevalence of attention deficit hyperactivity disorder (ADHD) diagnoses, 2007-2011 ${ }^{1}$

\begin{tabular}{lrrrrr}
\hline \multirow{2}{*}{ Category } & \multicolumn{5}{c}{ Total patients with ADHD } \\
\cline { 2 - 5 } & \multicolumn{1}{c}{2007} & 2008 & 2009 & 2010 & 2011 \\
\hline Total $(\mathrm{n})$ & 72,704 & 68,440 & 73,963 & 76,308 & 85,468 \\
$\quad$ Crude prevalence (\%) & 0.71 & 0.69 & 0.76 & 0.81 & 0.93 \\
$\quad$ Age-standardized prevalence (\%) & 0.67 & 0.67 & 0.75 & 0.94 \\
Gender & & & & \\
$\quad$ Boys & $58,005(1.07)$ & $54,684(1.05)$ & $59,160(1.17)$ & $60,899(1.24)$ & $67,487(1.41)$ \\
Girls & $14,699(0.30)$ & $13,756(0.29)$ & $14,803(0.32)$ & $15,409(0.34)$ & $17,981(0.41)$ \\
Age (yr) & $10.41 \pm 3.18$ & $10.69 \pm 3.16$ & $10.94 \pm 3.14$ & $11.04 \pm 3.19$ & $11.19 \pm 3.20$ \\
Age group (yr) & & & & & \\
1-5 & $2,944(0.13)$ & $2,445(0.11)$ & $2,151(0.10)$ & $2,215(0.10)$ & $2,449(0.11)$ \\
6-12 & $49,444(1.09)$ & $45,018(1.09)$ & $47,148(1.20)$ & $47,631(1.28)$ & $51,606(1.45)$ \\
13-17 & $20,316(0.58)$ & $20,977(0.59)$ & $24,664(0.71)$ & $26,462(0.77)$ & $31,413(0.94)$ \\
\hline
\end{tabular}

Values are presented as number (\%) or mean \pm standard deviation.

${ }^{1} \mathrm{ADHD}$ prevalence was calculated using Korean census statistics as the denominator.

${ }^{2}$ Age-standardized prevalence was estimated using the direct-standardization method with the 2010 standard population. 


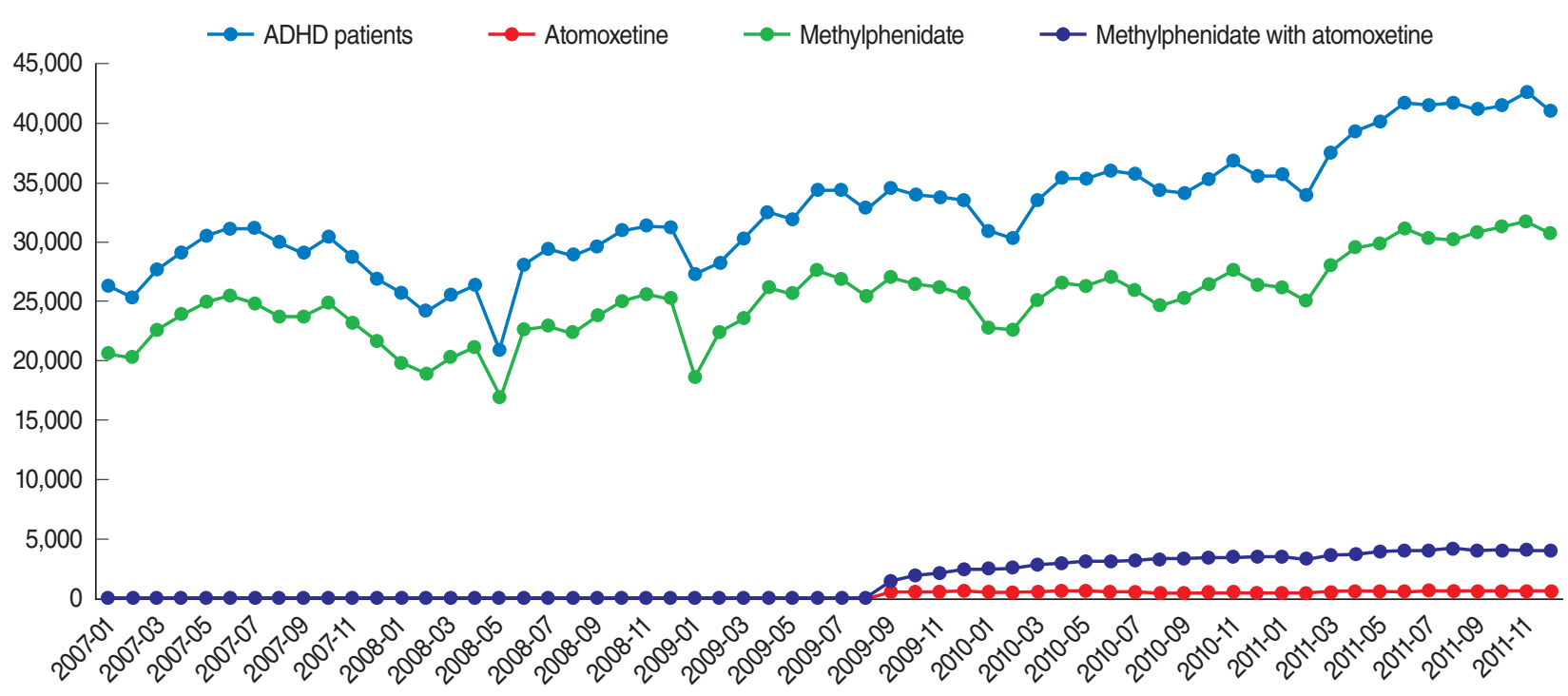

Figure 1. Time trends in attention deficit hyperactivity disorder (ADHD) diagnoses and use of methylphenidate and atomoxetine, $2007-2011$.

Moreover, the monthly number of ADHD diagnoses and patients prescribed medication showed generally upward trends, except for atomoxetine use (Figure 1). The majority of patients diagnosed with ADHD and treated with medication were boys. Over $70 \%$ of the patients diagnosed with ADHD were prescribed either methylphenidate or atomoxetine. The number of methylphenidate users increased from 53,739 in 2007 to 60,108 in 2011, whereas the number of atomoxetine users increased from 4,267 in 2009 to 11,190 in 2011. The proportion of methylphenidate users among ADHD patients decreased from $73.91 \%$ in 2007 to $70.33 \%$ in 2011 . The proportion decreased from $74.83 \%$ to $71.09 \%$ in boys and from $70.31 \%$ to $67.47 \%$ in girls. The proportion of atomoxetine users among ADHD patients increased from $5.77 \%$ in 2009 to $13.09 \%$ in 2011 . This proportion increased from $6.09 \%$ to $13.74 \%$ for boys and from $4.50 \%$ to $10.67 \%$ for girls. The proportion of methylphenidate users among patients aged 1-5 years decreased from $27.41 \%$ in 2007 to $7.59 \%$ in 2011. The number of prescriptions per patient increased from 6.85 in 2007 to 8.59 in 2011 for methylphenidate, while the number increased from 3.63 in 2009 to 6.87 in 2011 for atomoxetine. The number of prescribed days of ADHD medication per patient increased from 143.73 days in 2007 to 177.11 days in 2011 for methylphenidate, whereas it increased from 66.78 days in 2009 to 142.19 days in 2011 for atomoxetine (Table 2).

\section{DISCUSSION}

This study showed that less than $1 \%$ of children and adolescents in Korea were diagnosed with ADHD in 2007-2011. A recent study in Korea also showed that the prevalence of ADHD diagnosis and medication use in the pediatric population aged 6-18 years during $2008-2011$ was $0.799 \%$ and $0.610 \%$, respectively [7]. Current and previous studies suggest that the prevalence of ADHD diagnoses in Korea is low compared with other countries. Among US children and adolescents aged 4 to 17 years, the parent-reported prevalence of ADHD in 2011 was $8.8 \%$, and the prevalence of pharmacologically treated ADHD was $6.1 \%$, with the latter having increased by $28 \%$ from 2007 [1]. The prevalence of ADHD diagnosis and medication use in Canadian children was around 3\% [8]. The prevalence of ADHD diagnoses in Korea was on par with that in Hong Kong, where the prevalence of children on medication was $1.027 \%$ in 2013 [4]. The presence of differences among countries suggests that some children with ADHD are undiagnosed in Korea. This is especially likely given the finding of a $6.5 \%$ ADHD prevalence rate in a cross-sectional survey of elementary school children in Seoul [9].

The age-standardized annual prevalence of ADHD diagnoses in Korea increased substantially, from $0.67 \%$ in 2007 to $0.94 \%$ in 2011. Increasing trends have also been reported in many other countries. The rate of ADHD diagnosis in the US increased from $2.5 \%$ in 2001 to $3.1 \%$ in 2010 [10]. In the US pediatric population aged 0-17 years, ADHD prescriptions increased by $46 \%$ from 2002 to 2010 [2]. The prevalence of pharmacologically treated ADHD in the UK (per 1,000 persons) increased from 4.8 to 9.2 in children 6-12years of age and from 3.6 to 7.4 in those aged 13-17years from 2003 to 2008 [3]. The prevalence of children on ADHD medications in Hong Kong saw a 14-fold increase from 2001 to 2013 [4].

We found that the age structure of patients diagnosed with $\mathrm{ADHD}$ changed over time, with the mean age of patients be- 


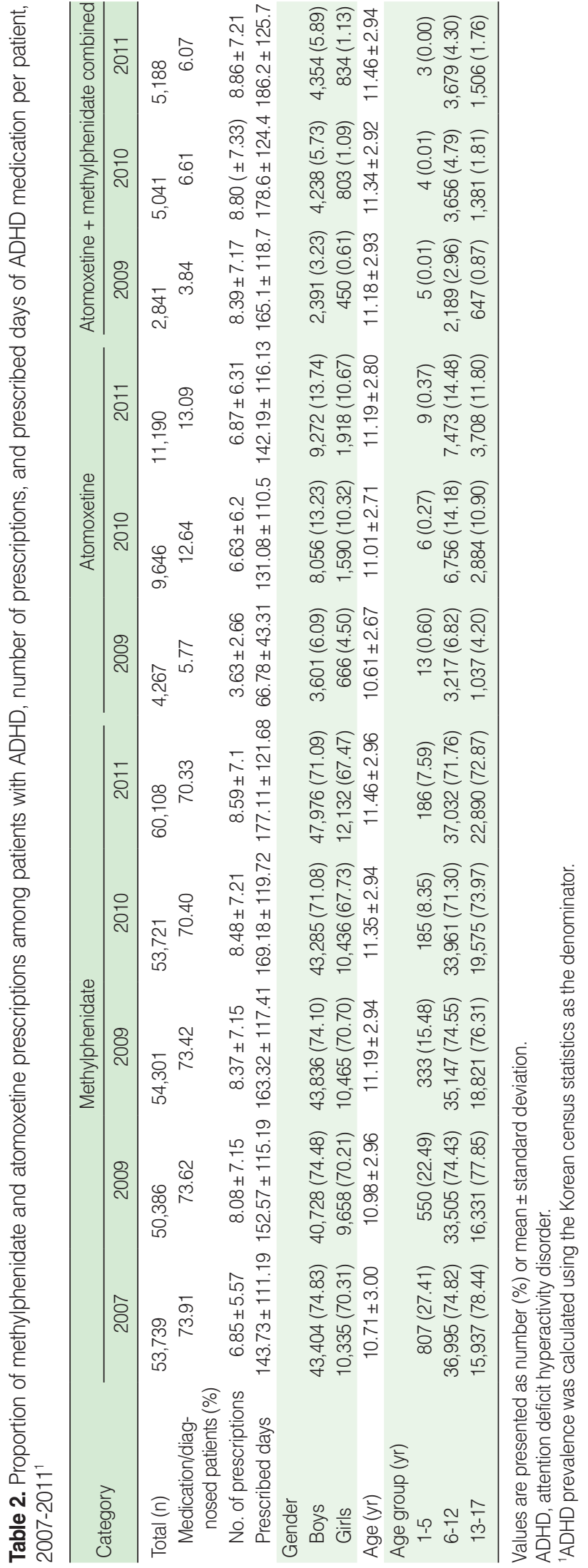

coming older.The number of patients aged 1-5 years decreased, while more patients aged 13-17 years were diagnosed with $\mathrm{AD}$ HD. Likewise, increases in the prevalence of ADHD in Canada were steeper among school-age children than among preschoolers between 1994 and 2007 [8].

A gender disparity in the prevalence of ADHD diagnosis and medication use was also noted in this study. Not only was the number of boys diagnosed with ADHD greater than that of girls, but the proportion of ADHD patients prescribed medication was also higher for boys than for girls. This is consistent with previous findings [9]. Nonetheless, our study indicated that the gender disparity decreased, because increases in ADHD prevalence and medication use were steeper among girls. Closing of the gender gap over time has been reported in the US [10] and Canada [8]. Additionally, the use of ADHD medications increased at a faster rate for female patients than for their male peers in the UK [3] and Hong Kong [4].

Along with the increasing prevalence of ADHD diagnoses over time, the number of prescriptions of ADHD medication per year and the number of prescribed days of ADHD medication per patient also increased.A prior study showed that $69 \%$ of newly diagnosed ADHD patients in Korea were pharmacologically treated and that methylphenidate was the most commonly used first medication [7]. Our data also indicated that over $70 \%$ of pediatric patients diagnosed with ADHD were prescribed methylphenidate, although its use showed a downward trend. The decrease in the number of methylphenidate users aged 1-5 years may be partially attributable to the announcement of the Korean regulatory body that the drug is contraindicated in this age group due to the risk of sudden cardiac death in December 2009 [11]. While the prevalence of methylphenidate users decreased, that of atomoxetine users increased, suggesting that some patients switched from methylphenidate to atomoxetine since the approval of the latter in Korea in 2009. It is worth noting that $6.61 \%$ of patients in our analysis used both methylphenidate and atomoxetine in 2010. In comparison, a Danish study showed that only $2 \%$ of methylphenidate prescriptions overlapped with atomoxetine treatment [12].

Our data suggest that a seasonal trend was present in ADHD diagnoses and the use of methylphenidate. This seasonal pattern appears to be attributable to school-year dosing in children; the number of diagnoses and methylphenidate users decreased in school-break months in the school calendar in Korea. However, similar seasonal variations were not observed for atomoxetine and combined ADHD drug users. It is plausible that our observed seasonal variation may be associated with the inappropriate prescribing of methylphenidate. Anecdotal experiences and/or perceptions of the incorrect belief that the use of methylphenidate may heighten a child's learning ability, thus improving test scores, may have contributed to the use of methylphe- 
nidate and the increase in $\mathrm{ADHD}$ diagnoses required for its prescription during spring and fall semester, followed by discontinuation during school breaks. It is possible that this phenomenon was observed only for methylphenidate, an ADHD drug that has been widely used since the 1990s, but not for atomoxetine, a relatively new drug.

A limitation of this study is that it used data on patients with ADHD obtained from the NHI database. It is highly likely that psychological disorders such as ADHD may be diagnosed and treated outside of the NHI system and paid for with out-of-pocket payments due to the stigma attached with the disorder.Therefore, the prevalence of ADHD diagnoses and medication use might have been underestimated in this study.

Additionally, the study subjects were limited to the population aged 17 years and younger, as most ADHD drugs approved by the Ministry of Food and Drug Safety are indicated for children and adolescents aged 6-17 years. However, as the prevalence of ADHD diagnoses has recently increased in adults and ADHD drug reimbursement has also been expanded to include adults (18-65 years) [13], future research is urgently needed to include all adolescents and adults in such studies using the latest data.

In conclusion, this study revealed that the prevalence of ADHD diagnoses and medication use in Korea increased from 2007 to 2011, although it remained relatively low compared to Western countries. The proportion of methylphenidate users among pediatric patients with ADHD decreased while the proportion of atomoxetine users increased. Future research is needed to examine comparative drug use patterns and how changes in the epidemiology of ADHD medication use affect patient safety.

\section{CONFLICT OF INTEREST}

The authors have no conflicts of interest to declare for this study.

\section{ORCID}

Inmyung Song http://orcid.org/0000-0001-7772-6617

Ju-Young Shin http://orcid.org/0000-0002-2791-1037

\section{REFERENCES}

1. Visser SN, Danielson ML, Bitsko RH, Holbrook JR, Kogan MD, Ghandour RM, et al. Trends in the parent-report of health care provider-diagnosed and medicated attention-deficit/hyperactivity disorder: United States, 2003-2011. J Am Acad Child Adolesc Psychiatry 2014;53:34-46.e2.

2. Chai G, Governale L, McMahon AW, Trinidad JP, Staffa J, Murphy D. Trends of outpatient prescription drug utilization in US children, 20022010. Pediatrics 2012;130:23-31.

3. McCarthy S, Wilton L, Murray ML, Hodgkins P, Asherson P, Wong IC. The epidemiology of pharmacologically treated attention deficit hyperactivity disorder (ADHD) in children, adolescents and adults in UK primary care. BMC Pediatr 2012;12:78.

4. Man KK, Ip P, Hsia Y, Chan EW, Chui CS, Lam MP, et al. ADHD drug prescribing trend is increasing among children and adolescents in Hong Kong. J Atten Disord 2014. doi: http://doi.org/10.1177/1087 054714536047 .

5. Bédard AC, Stein MA, Halperin JM, Krone B, Rajwan E, Newcorn JH. Differential impact of methylphenidate and atomoxetine on sustained attention in youth with attention-deficit/hyperactivity disorder. J Child Psychol Psychiatry 2015;56:40-48.

6. Du DT, Zhou EH, Goldsmith J, Nardinelli C, Hammad TA. Atomoxetine use during a period of FDA actions. Med Care 2012;50:987-992.

7. Hong M, Kwack YS, Joung YS, Lee SI, Kim B, Sohn SH, et al. Nationwide rate of attention-deficit hyperactivity disorder diagnosis and pharmacotherapy in Korea in 2008-2011. Asia Pac Psychiatry 2014; 6:379-385.

8. Brault MC, Lacourse É. Prevalence of prescribed attention-deficit hyperactivity disorder medications and diagnosis among Canadian preschoolers and school-age children: 1994-2007. Can J Psychiatry 2012;57:93-101.

9. Yang SJ, Cheong S, Hong SD. Prevalence and correlates of attention deficit hyperactivity disorder: school-based mental health services in Seoul. J Korean Neuropsychiatr Assoc 2006;45:69-76 (Korean).

10. Getahun D, Jacobsen SJ, Fassett MJ, Chen W, Demissie K, Rhoads GG. Recent trends in childhood attention-deficit/hyperactivity disorder. JAMA Pediatr 2013;167:282-288.

11. Shin JY, Lee SH, Shin SM, Shin HN, Park BJ. Regulatory action and moderate decrease in methylphenidate use among ADHD diagnosed patients aged five and under in Korea. Regul Toxicol Pharmacol 2015; 72:244-248.

12. Pottegård A, Bjerregaard BK, Glintborg D, Kortegaard LS, Hallas J, Moreno SI. The use of medication against attention deficit/hyperactivity disorder in Denmark: a drug use study from a patient perspective. Eur J Clin Pharmacol 2013;69:589-598.

13. Ministry of Health and Welfare. Drug reimbursement notice; 2016 Aug 25 [cited 2016 Oct 16]. Available from: http://www.mohw. go.kr/front_new/jb/sjb0406vw.jsp?PAR_MENU_ID=03\&MENU_ $\mathrm{ID}=030406 \&$ CONT_SEQ $=334054 \&$ page $=1$ (Korean). 